\title{
On the performance of metrics to predict quality in point cloud representations
}

\author{
Evangelos Alexiou and Touradj Ebrahimi \\ Multimedia Signal Processing Group (MMSPG) \\ École Polytechnique Fédérale de Lausanne (EPFL) \\ CH-1015, Lausanne, Switzerland
}

\begin{abstract}
Point clouds are a promising alternative for immersive representation of visual contents. Recently, an increased interest has been observed in the acquisition, processing and rendering of this modality. Although subjective and objective evaluations are critical in order to assess the visual quality of media content, they still remain open problems for point cloud representation. In this paper we focus our efforts on subjective quality assessment of point cloud geometry, subject to typical types of impairments such as noise corruption and compression-like distortions. In particular, we propose a subjective methodology that is closer to real-life scenarios of point cloud visualization. The performance of the state-of-the-art objective metrics is assessed by considering the subjective scores as the ground truth. Moreover, we investigate the impact of adopting different test methodologies by comparing them. Advantages and drawbacks of every approach are reported, based on statistical analysis. The results and conclusions of this work provide useful insights that could be considered in future experimentation.
\end{abstract}

Keywords: Point cloud, quality assessment, subjective methodologies, quality metrics

\section{INTRODUCTION}

The significant growth of 3D sensing technologies, along with the increased interest in adopting 3D representations in imaging systems, are shaping the way media content is created and used. In this environment, point clouds have become a viable solution, since they provide a practical way for capture, storage, delivery and rendering in augmented reality, mixed reality, virtual reality, medical imaging and 3D printing applications, among others. A point cloud can be defined as a collection of three-dimensional points in space representing the external surface of an object. Each sample is defined by its position, which is obtained by the measured or reconstructed X, $\mathrm{Y}$, and $\mathrm{Z}$ coordinates. Associated features can also be used in conjunction with the coordinate data to provide further information, such as the point's color, intensity, normal, size, curvature, and specularity.

Business and technology specialists predict that immersive applications will become mainstream by $2020 .^{1}$ Point clouds and mesh representations are expected to be modalities that will be extensively exploited, since they allow users to visualize static or dynamic contents in a more natural and immersive way. Although polygon mesh has been the prevailing form in computer graphics for 3D representations, point cloud is expected to be the future solution in real-time applications due to several important features: (a) High resolution point clouds can be directly acquired nowadays using professional or even consumer devices (e.g., Kinect v1, ${ }^{2}$ Kinect v2*, Intel RealSense $\left.{ }^{\dagger}, \mathrm{MC}^{3} \mathrm{D}^{3}\right)$. In the near future, conventional cameras are expected to be replaced by RGB-D cameras, since depth information can be simultaneously provided at approximately the same cost. (b) Polygon meshes require sophisticated reconstruction techniques when updating the content for every additional collection of points, with significant cost in terms of time and computational complexity. ${ }^{4-8}$ Using point clouds, the captured objects could be updated directly by rendering the additional points. (c) Compared to the meshbased compression algorithms, ${ }^{9-13}$ point clouds do not require storage, delivery and decoding of connectivity

\footnotetext{
Further author information: (Send correspondence to authors) E-mail: firstname.lastname@epfl.ch

*http://www.xbox.com/en-US/xbox-one/accessories/kinect

${ }^{\dagger}$ https://software.intel.com/en-us/realsense/home
} 
information (i.e., mesh topology and faces). The conversion to mesh representation for coding or rendering can also be avoided in many situations.

For these reasons, it is clear that point clouds will be extensively used in immersive communications in the near future. Visual quality and user experience of such emerging modalities play an important factor in how the content is acquired, transmitted and visualized. Hence, quality assessment should be investigated in a throrough manner. In general, quality evaluation is implemented via either objective or subjective assessments. Objective quality assessment is performed through computer algorithms that are designed to estimate signal distortions of a content. Typically, scores that correspond to human quality judgements are obtained. Subjective quality assessment is commonly conducted based on the ITU-R BT.500 recommendation ${ }^{14}$ and involves the participation of subjects in experiments in which distorted objects are visualized and their quality is rated. Such experiments are expensive in terms of both cost and time and, thus, objective quality metrics are frequently used instead. However, in order to get meaningful objective scores that can accurately predict human judgements, objective quality metrics have to be properly calibrated with respect to the subjective ratings, which are considered as the ground truth.

In the case of point clouds, quality assessment is still an open problem. As it provides a richer representation when compared to tradional media, traditional methodologies that were used until now in 2D imagery may not be suitable. Our approach on addressing subjective quality assessment of point cloud geometry is by adopting traditional methodologies, which are extended by involving interactivity between the user and the content in different experimental setups (i.e., environment and equipment). A double-stimulus impairement scale (DSIS) methodology has already been proposed under two different experimental frameworks; that is, a typical desktop setup and a head-mounted display in an augmented reality scenario. ${ }^{15,16}$ Although DSIS is preferred for its high accuracy and reliability in constructing a scale of perceptual references, it does not necessarily correspond to a realistic application where no reference of the distorted object is provided. In particular, it is substantially different when both the reference and the processed contents are visualized simultaneously, since the subjects typically rate based on relative geometrical differences that can be easily observed.

In this paper we extend our efforts on quality assessment of point cloud geometry by proposing the use of absolute category rating (ACR) of content impairement, as it consists of a methodology that is closer to real-life visualization of point clouds. We compare the results of subjective assessments of visual quality obtained by this methodology, with the results obtained by using DSIS methodology which was proposed in our previous work. ${ }^{15}$ This way we aim to address whether the subjects are able to perceive distortions in the same way when the reference object is absent and, more generally, what is the impact of adopting different methodologies in subjective quality assessment of point cloud geometry. In both cases, the experimental setup involves a typical desktop arrangement in a controlled environment through which the subjects are able to visualize, interact and rate the level of impairment of point cloud geometry after introducing typical degradations, such as noise and compression-like distortions. Identical contents, degradation values and equipment are used. Finally, the subjective scores are correlated with the state-of-the-art objective metrics.

\section{RELATED WORK}

A limited amount of work on subjective quality assessment of point clouds has been reported in the literature. Zhang et al. ${ }^{17}$ conducted experiments to subjectively assess colored point clouds for different geometric resolutions (i.e., downsampled versions of the original point cloud), and different values of geometric and color noise. The uniform noise that is applied, though, does not correspond to a realistic model of noise for point clouds, neither for geometric nor for color degradations. A typical desktop setup was used for experiments. However, it is not clearly reported by the authors whether the evaluation was passive or interactive and what was the adopted subjective methodolgy (i.e., ACR, DSIS, etc.). Mekuria et al. ${ }^{18}$ proposed a 3D tele-immersive system in which users represented by avatars (i.e., 3D point clouds captured by multiple Microsoft Kinect sensors) were able to interact in a virtual (i.e., synthetic) room, enabling a mixed reality scenario. The subjects were able to control the interaction of their avatar with the virtual environment through the use of the mouse in a desktop setup. The quality assessment of the dynamic colored point cloud (i.e., the navigavable avatar), was only a part of this complex experiment, whose main goal was to evaluate the efficiency of the proposed codec; that is, to subjectively assess the proposed compression algorithm in terms of distortions. In both cases, though, point 
cloud quality is not explicitly assessed. Furthermore, the point-to-plane metric is not taken under consideration and the correlation between objective and subjective metrics is not reported.

Javaheri et al. ${ }^{19}$ performed quality assessment of point cloud denoising algorithms. The authors use impulse noise (i.e., Gaussian noise with zero mean and large standard deviation) to simulate outlier errors. Two outlier removal algorithms (i.e., radius and sparse outlier removal) were tested with the first being adopted for the subsequent expermental procedure, as it was better correlated with the point-to-point objective metrics. After this pre-processing step, different amount of Gaussian noise was added to the processed models to mimic different levels of sensor imprecisions. Two denoising algorithms, namely Tikhonov regularization and total variation regularization were then applied on each of the degraded contents and subjectively assessed in a passive way. To visualize the content, the Poisson surface reconstruction was used and the resulted 3D object was captured by different viewpoints with a specific pattern to form a video. A continuous double-stimulus impairement scale protocol was adopted. The subjective scores were correlated with state-of-the-art objective metrics. However, as it is clearly stated, the scope of this paper was to subjectively assess denoising algorithms for point clouds, rather than the quality of the content. Furthermore, although the pre-processing steps followed before the visualization of the content could be considered realistic, their exact influence on the object under assessment is not evaluated and, thus, it is unknown. The impact of pre-processing is rather critical especially after applying a surface reconstruction technique, which typically changes the exact position and the number of points of the distorted point cloud to fit the underlying surface. This methodology, also, leads to assessment of meshes instead of point clouds. Finally, the passive subjective assessment protocol that is followed sounds interesting since the subjects are exposed to identical information regarding the content under evaluation; however, interactivity is a desirable and natural characteristic when it comes to immersive content which is not exploited.

In, ${ }^{15,16}$ we proposed an interactive subjective evaluation of point cloud geometry using a typical desktop setup and a head-mounted display in an augmented reality scenario, respectively. In both cases, two different types of distortions were evaluated, namely Gaussian noise and octree-pruning, to simulate position errors from sensor inaccuracies and compression-like artifacts, correspondingly. Simultaneous DSIS subjective methodology was adopted and, thus, the subjects were assessing the level of impairment, after visualizing and interacting with both the original and the processed contents simultaneously. Although this protocol is preferred for its high accuracy, it does not correspond to a realistic application of visualizing point clouds. In this study, we adopt the ACR content impairement methodology and we compare it against the DSIS test method. Furthermore, the performance of the objective metrics is assessed against the subjective ratings.

\section{SUBJECTIVE EXPERIMENTS}

This section reports how the subjective quality evaluation experiments were designed. Specifically, the creation of the contents, the adopted distortions, the equipment and the testing environment are described.

\subsection{Selection of contents}

In this study, subjective quality assessment of point cloud geometry is performed in a typical desktop setup. The point clouds are displayed without using any rendering algorithm or surface reconstruction technique; that is, a collection of points is rendered in a flat screen. In order to assess only the geometrical errors, no color values were assigned to the points since the presence of additional attributes can be considered distracting. For this reason five simple objects were selected, since it would be difficult for complex scenes or models to be distinguishable in the absence of color. In addition, increasing the complexity of the object would lead to more uncertainty in the exact representation of the underlying object, since the underlying surface is practically reconstructed by internal cognitive processess that takes place in the human brain. Moreover, the contents were scaled to be fitted in a minimum bounding box of size 1 in order to normalize the impact of distortions. Finally, they were selected so as to be diverse in terms of geometrical structure.

In Figure 1 the selected contents are presented, while in Table 1 the corresponding number of points, minimum and maximum Nearest Neighbour (NN) distances (i.e., intrinsic resolutions), and the dimensions of the minimum bounding box across $\mathrm{X}, \mathrm{Y}$ and $\mathrm{Z}$ axes are provided. Cube and sphere are artificially generated point clouds and represent synthetic contents with perfect geometry. Vase is a model captured by Intel RealSense R200 and constitutes a representative irregular point cloud that can be acquired from a low-cost consumer market device. 
Finally, bunny and dragon are selected from the Stanford 3D Scanning Repository ${ }^{\ddagger}$ and represent less irregular contents with very limited amount of noise and smooth underlying surfaces.

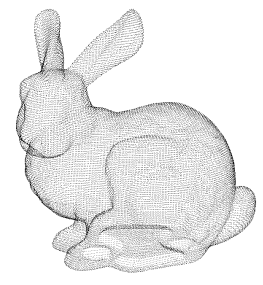

(a) Bunny

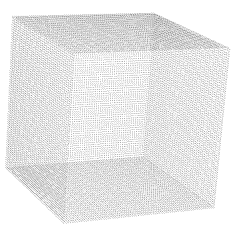

(b) Cube

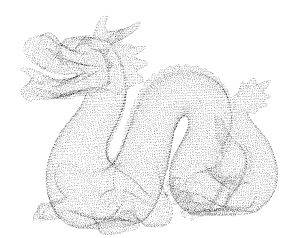

(c) Dragon

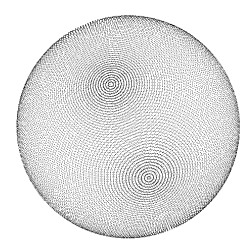

(d) Sphere

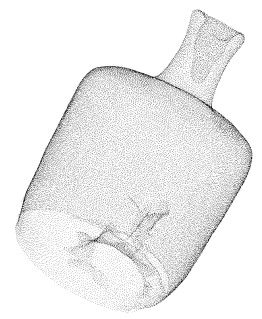

(e) Vase

Figure 1: Selected contents

It should be mentioned that in order to eliminate the impact of highly diverse contents in terms of density, a sparser version of the dragon was used (i.e., namely, dragon_vrip_res3), and the initially captured vase was downsampled using CloudCompare ${ }^{20}$ software. For the former case, a minimal distance between two points was set to a specific value, ensuring that no point in the output content is closer to another point than the specified value. Furthermore, no displacements in the original coordinates of the points was introduced, maintaining the default irregular structure of this content.

Table 1: Number of points and intristic resolutions per content

\begin{tabular}{c|c|c|c|c|c} 
Contents: & Bunny & Cube & Dragon & Sphere & Vase \\
\hline Points: & 35947 & 30246 & 22998 & 30135 & 36022 \\
Minimum NN: & 0.000038 & 0.010051 & 0.000758 & 0.000148 & 0.005501 \\
Maximum NN: & 0.014384 & 0.014142 & 0.011309 & 0.012825 & 0.010421 \\
Dimensions X/Y Z: Z: & $1 / 0.99 / 0.78$ & $1 / 1 / 1$ & $1 / 0.71 / 0.45$ & $1 / 1 / 1$ & $0.68 / 1 / 0.67$
\end{tabular}

\subsection{Degradation types}

In this experiment, two different types of geometrical degradations are assessed: (a) noise, which results in position errors after points' displacement according to the selected distribution, and (b) octree-pruning, which leads to a sparser and more regular version of the original point cloud, obtained after applying an octree structure with appropriate level-of-detail (LoD) values. Thus, in the latter case, the processed content is subject to both points' displacement and points' removal. The distortion values in both cases were selected so as to cover a wide range of subjective scores (i.e., from the lowest to the highest) for both types of distortions.

\subsubsection{Noise}

Gaussian noise is widely used in the literature to model position errors due to sensor imperfections during acquisition, or after stereoscopic triangulation which can be performed either in real-time using more than one synchronized cameras, or offline by using images of the same scene taken from different viewpoints. In our case, we assume that the noise affects the position of all points of the point cloud and its level is determined by a target standard deviation (i.e., $\sigma=\{0.0005,0.002,0.008,0.016\}$ ).

\subsubsection{Octree-pruning}

Octree structure is extensively exploited in point cloud compression algorithms, as it enables an efficient way for regular representations of points with known maximum geometrical error. It is the basis of many compression algorthms that are currently used, and it is further exploited to reduce the size of data needed for content reconstruction..$^{21-25}$ This regular representation, though, leads to visible artifacts in the form of structured loss. Octree-pruning can be obtained by setting a desirable octree LoD, which defines the size of leaf nodes.

\footnotetext{
${ }^{\ddagger} \mathrm{http}: / /$ graphics.stanford.edu/data/3Dscanrep/
} 
A minimum bounding box encloses the content and, in each level, each box is sub-divided into 8 smaller and equally sized boxes. A point can be appended only in leaf nodes and all the points that are enclosed in a leaf node are collectively represented by the center of that node. Thus, octree stucture leads to points' removal and displacement, while the error is limited by the diagonal of the leaf node divided by two. By increasing the LoD, the number of points of the processed content obviously decreases. In our case, LoD values are selected for each content in order to obtain target percentages $(p)$, with respect to the original number of points, with an acceptable deviation of $\pm 2 \%$ (i.e., $p=\{30 \%, 50 \%, 70 \%, 90 \%\}$ ).

\subsection{Environment and equipment}

The experiments were conducted in the MMSPG laboratory which fulfills the recommendations for subjective evaluation of visual data issued by ITU. ${ }^{14}$ PCLVisualizer ${ }^{26}$ with an Apple Cinema Display of 30 inch (res. 2560x1600) was used to display the stimuli. The background of the environment was set to black and the color of the point cloud was set to white. The luminance of the points and the background was measured on the flat screen with a luminosity sensor ${ }^{\S}$ providing the values of 354 and 0.5 nits, respectively. The subjects were able to visualize the point clouds on the flat screen, interact with them using the mouse (left, right, up, down, zoom), and provide their scores using the keyboard.

\subsection{Subjective evaluation methodologies}

The ACR with 5-scale impairment rating test methodology was selected in order to (a) perform quality assessment adopting a more realistic type of point cloud visualization, and (b) assess the impact of adopting different subjective methodologies in point cloud geometry evaluation. As the selected degradation values for the Gaussian noise and the sparsity level of dragon and vase were modified with respect to our previous work, ${ }^{15}$ as reported in Section 3.1, we also re-ran the experiments adopting the DSIS test methodology and using the updated contents.

Since two different types of degradations were assessed, the evaluation procedure of each test methodology was split in two different sessions (i.e., 4 sessions in total: DSIS with Gaussian noise, DSIS with octree-pruning, ACR with Gaussian noise, and ACR with octree-pruning). Each session was launched after a training phase, where special care was given in order for the subjects to well understand the impact of each type of impairment. During training, the subjects also familiarized with the interaction part.

In the DSIS test method, participants were instructed to rate the level of impairment of the processed content with respect to the reference, which was always visible. The users always knew the position of both contents, since the stimuli were annotated with text in the visualization software. In the ACR experiment, the subjects were asked to rate the level of impairment of the processed content with respect to their internal reference. In both cases, the subjects were free to interact with the content and change the angle of view according to their preference. No time limitation was set before providing their scores.

In both ACR and DSIS experiments, different permutations of the order of visualization content was applied per subject and session. In addition, to reduce contextual effects in the DSIS method, the position of the reference while remaining fixed across each session for every subject, changed randomly for different subjects. Finally, the same content was never displayed consecutively.

A total of 5 contents and 4 degradation values were used, along with a hidden reference. This leads to 25 stimuli per session. A total of 28 naive subjects (17 males and 11 females) participated in the experiments; 12 of them were involved in both experiments while 16 participated in just one, leading to 20 scores per stimulus. The age was ranging from 20.56 to 37.4 with an average of 28.18 and a median of 28.04 years old.

\section{STATISTICAL ANALYSIS}

In this section the statistics computed from the subjective scores are reported, followed by an overview of the state-of-the-art objective metrics. Then, a description of the performance indexes used to assess the objective metrics is provided. Finally, the statistics to compare the subjective methodologies are described.

\footnotetext{
${ }^{\S}$ X-Rite i1 Display Pro - http://www.xrite.com/
} 


\subsection{Subjective quality metrics}

For each session and each experiment, outlier detection and removal was performed on the subjective scores following the Recommendation ITU-T P.1401. ${ }^{27}$ In the ACR experiment, no outlier was detected in none of the two sessions (i.e., Gaussian noise and octree-pruning) leading to 20 out of the 20 scores for both cases, while in the DSIS experiment one outlier was detected in the second session leading to 20 out of 20 and 19 out of 20 scores for Gaussian noise and octree-pruning, respectively. After outlier removal, the mean opinion score (MOS) was computed for each degraded content, along with the corresponding $95 \%$ confidence intervals assuming a Student's t-distribution.

\subsection{Objective quality metrics}

In objective quality assessment of point clouds, full-reference metrics are used and are based on the relative geometrical differences between the points of the processed and the reference contents. In other terms, geometrical similarity is the key factor. The state-of-the-art objective metrics for geometric distortions can be classified as point-to-point (p2point) and point-to-plane (p2plane). ${ }^{18,28}$ In the first case, p2point error is calculated by connecting each point of the content under evaluation to the closest point that belongs to the reference point cloud. In the second case, the normal of each point of the reference point cloud should be given or estimated. Then, the p2plane error of a point of a content under evaluation is calculated by measuring the projected error along the normal of the closest point that belongs to the reference point cloud. This applies when both stimuli have identical number of points. Otherwise, every point of the content under evaluation is associated with a non-overlapping nearest neighborhood of points that belongs to the reference. Then, an average normal vector of the points classified into the same neighborhood is computed. Finally, the p2plane error of a point under assessment is calculated by measuring the projected error along the associated averaged normal vector.

Geometric errors between the original and the processed point clouds can be estimated either using the Root Mean Square (RMS) difference, the Mean Squared Error (MSE), or the Hausdorff ${ }^{\mathbb{I}}$ distance for both p2point and p2plane cases. It is rather common to use the symmetric distance, which is obtained by setting both the original and the processed content as reference and estimating the absolute values of error in both cases. Then, the maximum value is considered as the error. ${ }^{18}$ However, such absolute values do not provide meaningful results when contents of different dimensions are assessed. For this purpose, the Peak-to-Signal Noise Ratio (PSNR) is proposed. In the literature, it is defined as the ratio of the squared maximum distance of the nearest neighbours (potentially, multiplied by a scalar), or the squared distance of the diagonal of the minimum bounding box divided by the squared error value (i.e., MSE, squared RMS or squared Hausdorff). For the sake of completeness, we should also mention that the color distortion of a point cloud is commonly assessed using the well-know PSNR ratio used in conventional 2D images; that is the maximum color value divided by the mean squared error.

In this study, the version 0.02 of the software described $i^{28}$ is used. In particular, the RMS difference and the Hausdorff distance are considered for both cases of p2point and p2plane metrics. To compute the p2plane error, the normals of each content are estimated based on principal component analysis considering 12-nearest neighbours around each point. The PSNR is defined as the ratio of the squared maximum distance of the nearest neighbours divided by the squared error value. After evaluating the error by setting both the original and the processed content as a reference, the symmetrical distance is considered as the final error value. This leads to a total of 8 different objective metrics, for which performance is assessed with respect to the subjective scores of both testing methodologies.

\subsection{Performance indexes for objective metrics}

The objective metrics are benchmarked with respect to the subjective MOS, which are considered as the ground truth. The result of execution of a particular objective metric indicates a Point cloud Quality Rating (PQR). A predicted MOS, denoted as $\mathrm{MOS}_{P}$ which corresponds to a specific distorted content, is estimated by applying a fitting function to each [PQR, MOS] pair. According to Recommendation ITU-T P.1401, ${ }^{27}$ the following properties of the PQR are considered: linearity, monotonicity, accuracy and consistency. They are obtained

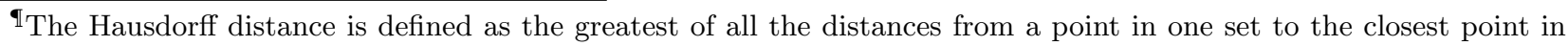
the other set.
} 
by computing the Pearson linear correlation coefficient (PCC), the Spearman rank order correlation coefficient

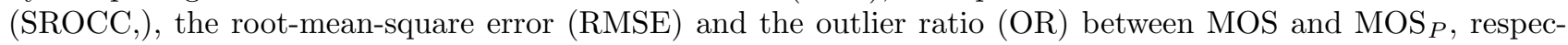
tively. Linear, logistic and cubic fittings were tested and it was found that the latter provides the best results. Hence, the cubic fitting is used to demonstrate the performance indexes results.

\subsection{Comparison between subjective methodologies}

In this experiment two different types of degradations are adopted. The comparison between subjective methdologies, as described below, is performed per degradation type, as the visible distortions in the processed point clouds from these types of impairments are radically different. This, essentially, means that we compare ACR against DSIS after introducing Gaussian noise, and ACR against DSIS after octree-pruning, separately.

According to Recommendation ITU-T P.1401, ${ }^{27}$ to compare two different testing methodologies, several fittings were applied to the obtained MOS values. In particular, no fitting, linear, and cubic fitting were tested. The PCC, SROCC, RMSE and OR were also computed to account for linearity, monotonicity, accuracy and consistency, respectively. Finally, correct estimation (CE), under estimation (UE) and over estimation (OE) percentages were determined after a multiple comparison test at a 5\% significance level to check whether both methodologies give statistically equivalent MOS results. CE is obtained when the MOS of a data point obtained from the first methodology (i.e., ground truth) lies within the confidence interval estimated using the second methodology for this content, and vice versa. UE occurs when the MOS of a data point obtained from the second methodology is lower than the lower bound of the confidence interval estimated for this content from the first methodology. OE occurs when the MOS of a data point obtained from the second methodology is higher than the upper bound of the confidence interval estimated from the first methodology.

The classification errors, namely false ranking (FR), false differentiation (FD) and false tie (FT), were also computed along with correct decision (CD), to check whether the subjective methodologies lead to different conclusions on a pair of data points. ${ }^{29}$ A multiple comparison test at a $5 \%$ significance level was performed to determine when two data points are statistically equivalent, and when they are statistically distinguishable. Then, for each pair of data points (A, B), there are three possible categories: (i) A is better than B, (ii) A is the same as B, and (iii) A is worse than B. This three-way classification is performed for both subjective methodologies. When both test methods agree, a CD occurs. When the first subjective methodology (i.e., ground truth) says that $\mathrm{A}$ is better than $\mathrm{B}$, or $\mathrm{A}$ is worse than $\mathrm{B}$, and the second subjective methodology says that A is the same as B, a FT occurs. This is the least offensive error. When the first subjective methodology says that $\mathrm{A}$ is the same as $\mathrm{B}$, and the second subjective methodology says that $\mathrm{A}$ is better than $\mathrm{B}$, or $\mathrm{A}$ is worse than B, a FD occurs. This is a more offensive error. Finally, when the first subjective methodology says that A is better than $\mathrm{B}$, or $\mathrm{A}$ is worse than $\mathrm{B}$, and the second subjective methodology says the opposite, a FR occurs. This is the most offensive type of error.

Finally, one-way analysis of variance (ANOVA) was performed to identify whether the subjective evaluation methodologies are statistically different. Additionally, multi-way ANOVA was performed on the raw scores of each type of distortion to further assess the influence of the selected parameters (i.e., degradation level, content) on the subjective results.

\section{RESULTS AND DISCUSSION}

In this section the performance of the objective metrics against the degradation levels for each type of distortion are provided. Then, the subjective scores as obtained from every evaluation methodology are presented, against the degradation levels. Moreover, the performance of the objective metrics is investigated per type of impairment and test method, considering as ground truth the corresponding subjective scores. Finally, a comparison is conducted between the adopted subjective methodologies based on a statistical analysis.

\subsection{Objective results}

In Figure 2 the error as computed by two out of the eight objective metrics is presented against the target standard deviation of Gaussian noise. In particular, in Figure 2a, the estimated geometric error using the p2point metric with Hausdorff distance is depicted, whilst in Figure 2b the PSNR values using the same metric 
and distance are presented, for every selected content. In Figure 3 the error as computed by two other objective metrics against the target percentage of remaining points after octree-pruning is demonstrated. Specifically, in Figure 3a, the geometric error using the p2plane metric with Hausdorff distance is presented, while in Figure 3b the corresponding PSNR values are provided, for all contents. As can be seen, the geometric errors occuring either after the displacement of the points due to Gaussian noise, or by decreasing the number of points after octree-pruning are captured by the objective metrics. Similar trends are observed in other objective metrics, for each type of impairment.

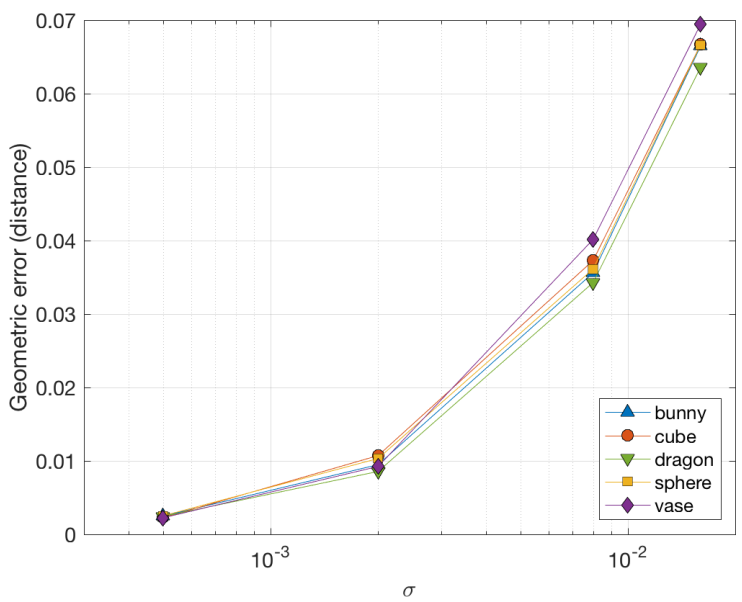

(a) p2point Hausdorff

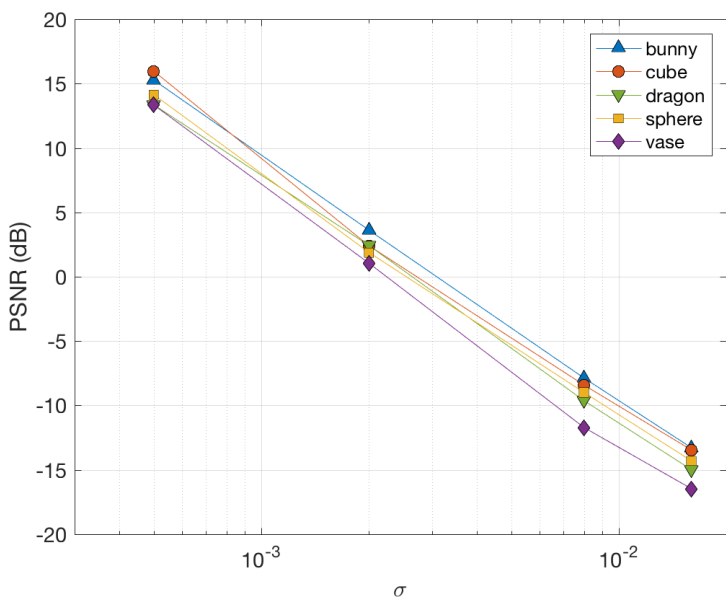

(b) PSNR - p2point Hausdorff

Figure 2: Objective scores against degradation values in the presence of Gaussian noise in a logarithmic scale.

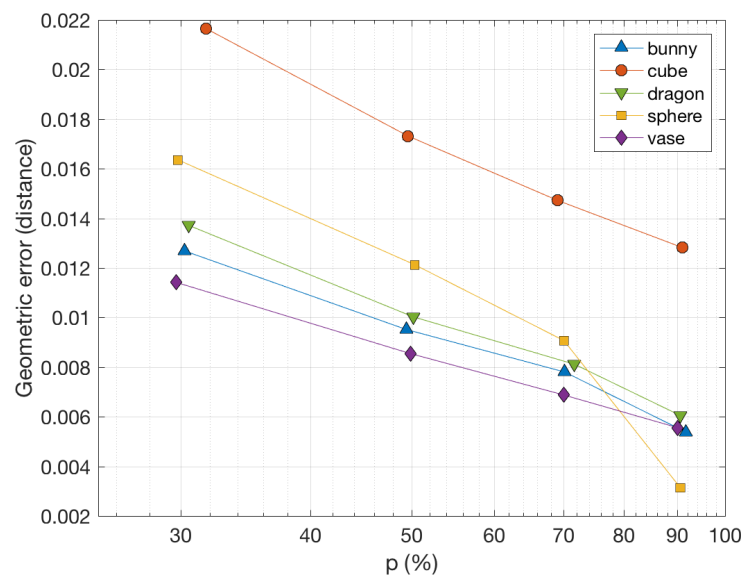

(a) p2plane Hausdorff

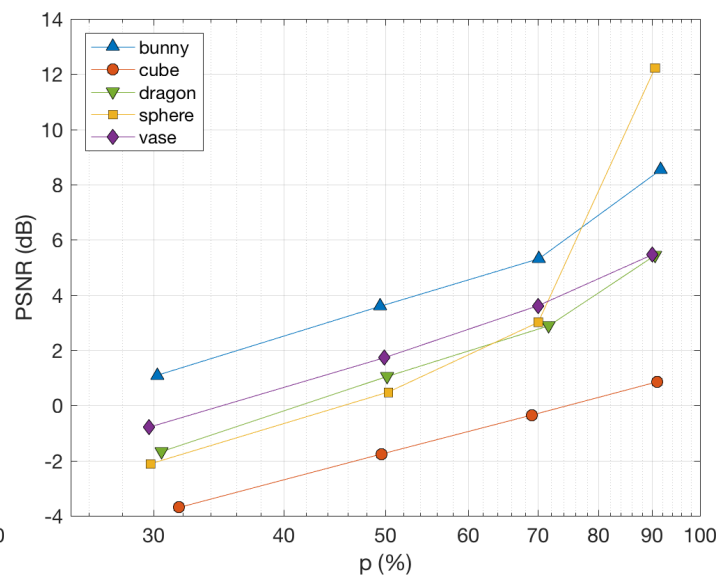

(b) PSNR - p2plane Hausdorff

Figure 3: Objective scores against degradation values after octree-pruning in a logarithmic scale.

\subsection{Subjective results}

In Figures 4 and 5 the MOS along with the confidence intervals against the degradation values of every type of impairment are presented for the DSIS and ACR experiments, respectively. The markers with faces indicate the scores for the distorted versions of the original point clouds, while the markers without faces (i.e., at the top-right of Figures 4a, 5a and top-left of Figures 4b, 5b) correspond to the scores of the hidden references.

Regarding the results obtained from the DSIS experiment, it is obvious that as the level of impairment is increasing, the MOS decreases, except in the case of dragon which is slighlty worse after octree-pruning for 


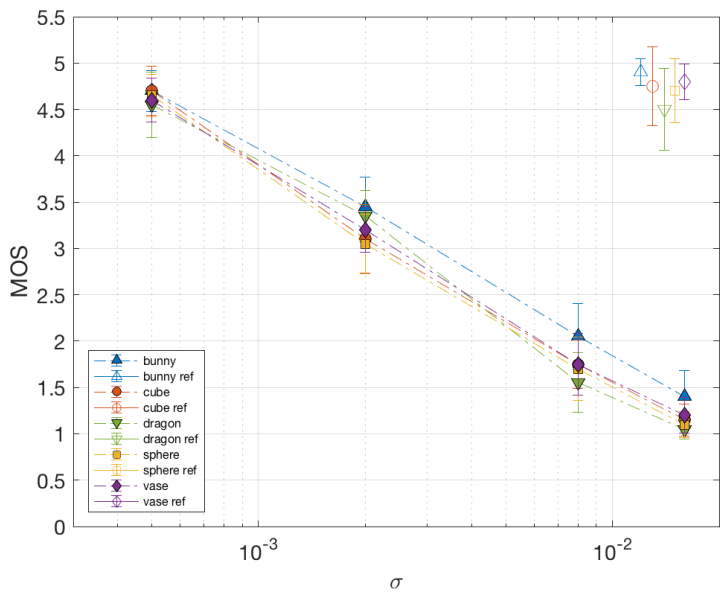

(a) Gaussian noise.

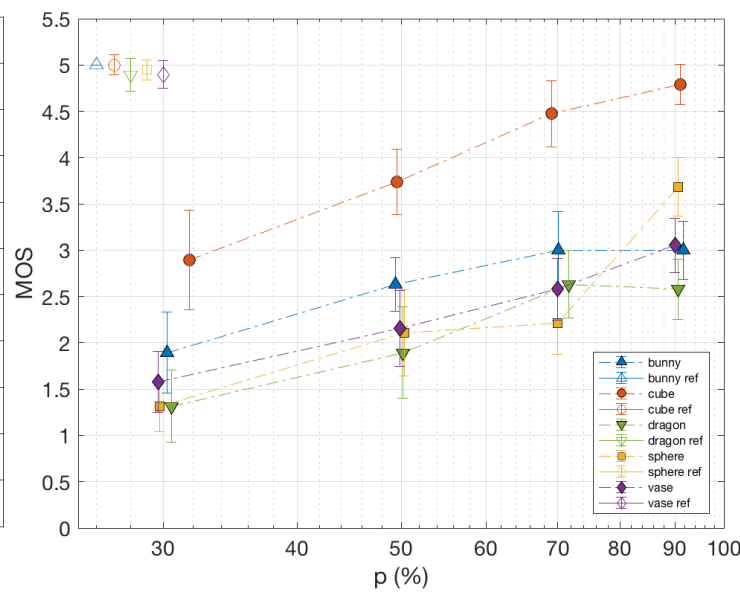

(b) Octree-pruning.

Figure 4: Subjective scores against degradation values adopting DSIS test method.

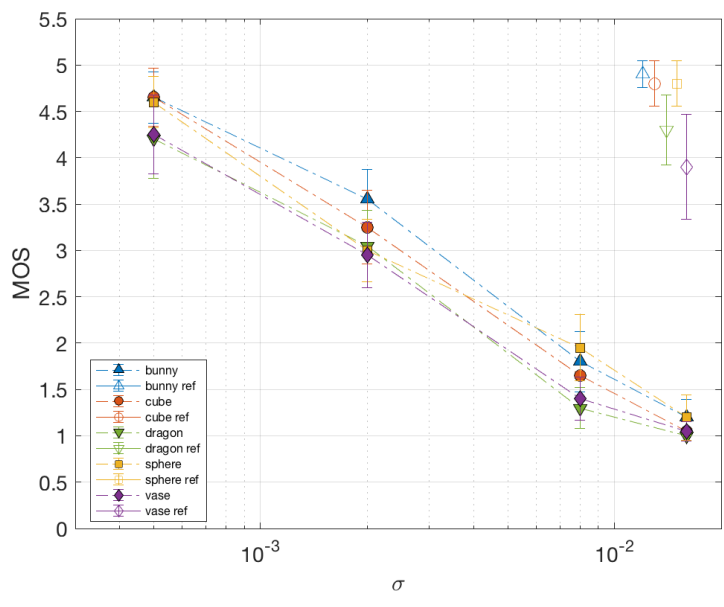

(a) Gaussian noise.

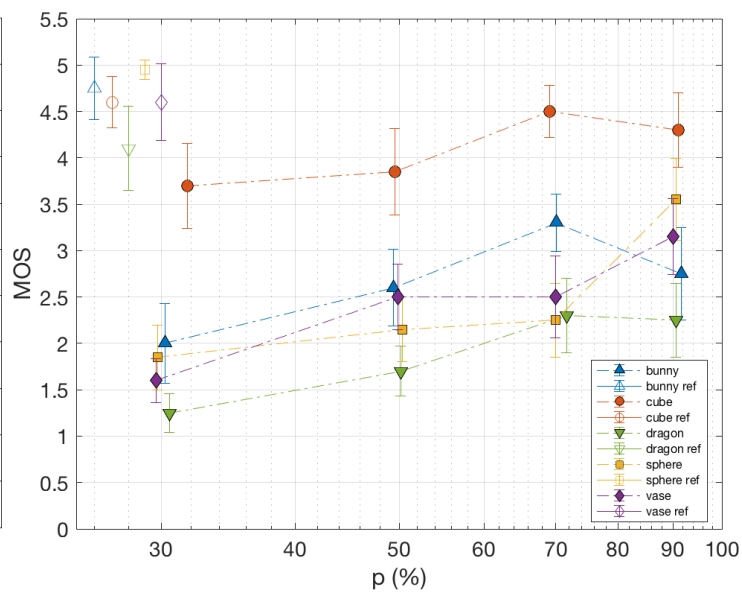

(b) Octree-pruning.

Figure 5: Subjective scores against degradation values adopting ACR test method.

$p=90 \%$ when compared to $p=70 \%$. In general, the subjects were able to recognize easily the level of distortion, since the reference content was always visible. In case of Gaussian noise, the subjective scores are decreasing following a logarithmic trend as the target standard deviation increases, for every type of content. The shape of the underlying surface of the content seems to be critical in the case of compression-like distortions. In particular, cube, which is the only content that consists of only planar surfaces, is rated remarkably higher than any other content, for every level of impairement.

Considering the results of the ACR experiment, generally, similar trends for both types of degradations are observed. The confidence intervals are larger in this case; however, this is expected as the subjects do not rate the level of impairement of the processed content with respect to the visible reference, but based on their internal reference. In the case of Gaussian noise, the dragon and vase are rated slightly but consistently lower than the other contents, for every target standard deviation value. This difference is even more observable in the case of the hidden references. For dragon, we assume that this behavior is observed due to the complexity of the object. In particular, although the density of all the contents is similar (i.e., in the same order of magnitude), the dragon is a substantially more complex object and, thus, a denser version would be preferred. This speculation could be partially verified by how the subjects rate the hidden reference after octree-pruning, which is lower than all the other contents; meaning that subjects tend to believe that the original content is perceived as being already simplified. Regarding vase, the difference in subjective scores is assumed to be observed due to its irregular 
geometrical structure. There are two main reasons for this: (a) due to the training phase, which was conducted involving a more regular content; thus, when irregular geometry was observed, participants tended to associate it with the existence of noise, and (b) due to a general preference of subjects to visualize regular point clouds. In the case of the octree-pruning, as the subjects expected structure loss which was not perceived, the hidden reference was rated similarly to the other original contents.

In the ACR experiment and considering the case of compression-like distortions, cube is again rated substantially higher than all other contents. Interestingly, in the ACR test method it is rated even higher than in the DSIS method for $p=30 \%$. Furthermore, a steep increase of the MOS of the sphere can be observed for $p=90 \%$. The same trend can be noticed also in the DSIS scores. Sphere was artificially generated and the distribution of points across its surface is not uniform. In particular, the density of points in the poles is much higher and, thus, for $p=90 \%$, no significant distortions are perceived in the remaining surface. This can be also observed in Figure 3, where a reduction in the amount of error captured by the objective metrics is notable. Finally, bunny is rated remarkably lower for $p=90 \%$ when compared to $p=70 \%$. This is because, indeed, additional artifacts are visible that reduce the perceptual quality of the content for $p=90 \%$. However, this trend is not that clear in the case of DSIS test method, although the scores are not better as anyone would expect. This leads to the assumption that subjects tend to rate based on the number of points of the processed point cloud when the reference content is provided. However, when just one point cloud is visualized, the visual quality is the only factor that is assessed. These obserations also suggest that simplifying objects without considering their underlying geometrical properties, or without using more sophisticated methods, even with more data, enhanced visual distortions may occur.

\subsection{Performance of the objective metrics}

In Tables 2 and 3, the performance indexes of the state-of-the-art objective metrics against the subjective scores are presented. In particular, Table 2 indicates the performance of objective metrics adopting the DSIS methodology, while Table 3, depicts their performance when adopting the ACR methodology. In both cases, both types of impairment are investigated separately.

Table 2: Performance indexes for the different objective metrics adopting the DSIS test method.

\begin{tabular}{|c|c|c|c|c|c|c|c|c|}
\hline \multirow{2}{*}{ Metric } & \multicolumn{4}{|c|}{ Gaussian noise } & \multicolumn{4}{|c|}{ Octree-pruning } \\
\hline & PCC & SROCC & RMSE & OR & $\mathrm{PCC}$ & SROCC & RMSE & OR \\
\hline $\mathrm{p} 2 \mathrm{p}$ & 0.9949 & 0.9488 & 0.1404 & 0.05 & 0.1524 & -0.1499 & 0.9403 & 0.70 \\
\hline & 9905 & 0.9 & 0.1904 & 0.10 & 0.1884 & -0. & 44 & 0.70 \\
\hline & 0.9936 & 0.9 & 0.1569 & 0.10 & 0.2473 & 0.2 & 0.9219 & 0.55 \\
\hline & 0.9931 & 0.9368 & 0.1622 & 0.10 & 0.2219 & 0.1695 & 0.9277 & 0.60 \\
\hline & 0.9948 & 0.9707 & 0.1416 & 0.10 & 0.2840 & 0.2765 & 0.9122 & 0.50 \\
\hline PS & 0.9947 & 0.9707 & 0.1424 & 0.05 & 0.2837 & 0.2328 & 0.9123 & 0.50 \\
\hline PSI & 0.9960 & 0.9782 & 0.1231 & 0.05 & 0.3364 & 0.4384 & 0.8960 & 0.60 \\
\hline PSNR - p2plane Hausdorff & 0.9959 & 0.9782 & 0.1251 & 0.05 & 0.2860 & 0.3119 & 0.9177 & 0.50 \\
\hline
\end{tabular}

Table 3: Performance indexes for the different objective metrics adopting the ACR test method.

\begin{tabular}{|c|c|c|c|c|c|c|c|c|}
\hline \multirow{2}{*}{ Metric } & \multicolumn{4}{|c|}{ Gaussian noise } & \multicolumn{4}{|c|}{ Octree-pruning } \\
\hline & $\mathrm{PCC}$ & SROCC & RMSE & OR & $\mathrm{PCC}$ & SROCC & RMSE & OR \\
\hline p2point $_{\text {BMS }}$ & 0.9867 & 0.9326 & 0.2232 & 0.25 & 0.3698 & -0.0045 & 0.8497 & 0.60 \\
\hline p2plane $\mathrm{RMS}$ & 0.9862 & 0.9439 & 0.2276 & 0.15 & 0.3985 & -0.0467 & 0.8388 & 0.60 \\
\hline p2point ${ }_{\text {Hausdorff }}$ & 0.9864 & 0.9304 & 0.2260 & 0.15 & 0.2540 & -0.0956 & 0.8846 & 0.60 \\
\hline p2plane ${ }_{\text {Hausdorff }}$ & 0.9871 & 0.9304 & 0.2201 & 0.20 & 0.3185 & -0.0211 & 0.8670 & 0.60 \\
\hline PSNR - p2point RMS & 0.9930 & 0.9846 & 0.1629 & 0.10 & 0.2207 & 0.1415 & 0.8920 & 0.65 \\
\hline PSNR - p2plane RMS & 0.9949 & 0.9846 & 0.1391 & 0.10 & 0.3059 & -0.0805 & 0.8707 & 0.60 \\
\hline PSNR - p2point ${ }_{\text {Hausdorff }}$ & 0.9945 & 0.9846 & 0.1435 & 0.15 & 0.2291 & 0.2942 & 0.8902 & 0.65 \\
\hline PSNR - p2plane Hausdorff & 0.9950 & 0.9846 & 0.1371 & 0.15 & 0.2202 & 0.1685 & 0.8921 & 0.65 \\
\hline
\end{tabular}


In Figures 6 and 7 scatter plots of the subjective scores against the most efficient objective metric are provided. In Figure 6, the MOS values are obtained from the DSIS test method. In Figure 7, the subjective scores from the ACR experiment are used. In both figures, the performance against all types of distortion is presented separately.

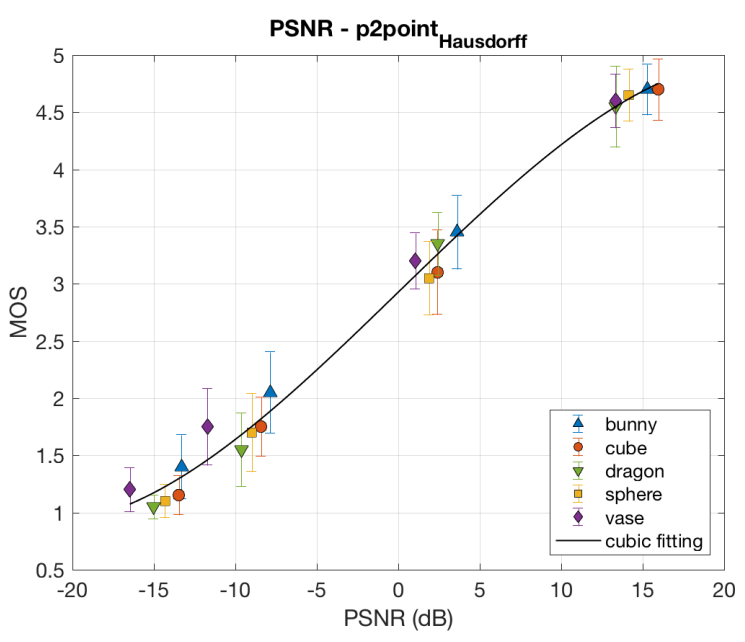

(a) Gaussian noise.

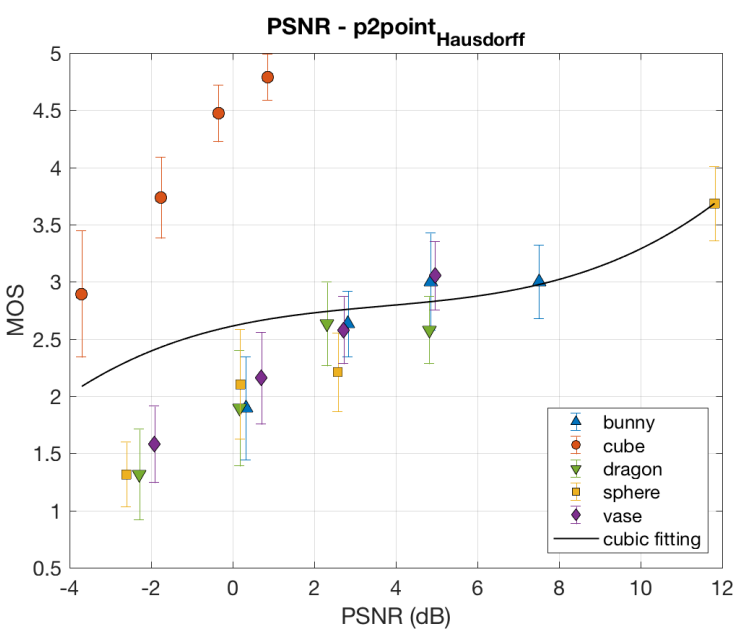

(b) Octree-pruning.

Figure 6: Subjective vs objective results adopting DSIS test method.

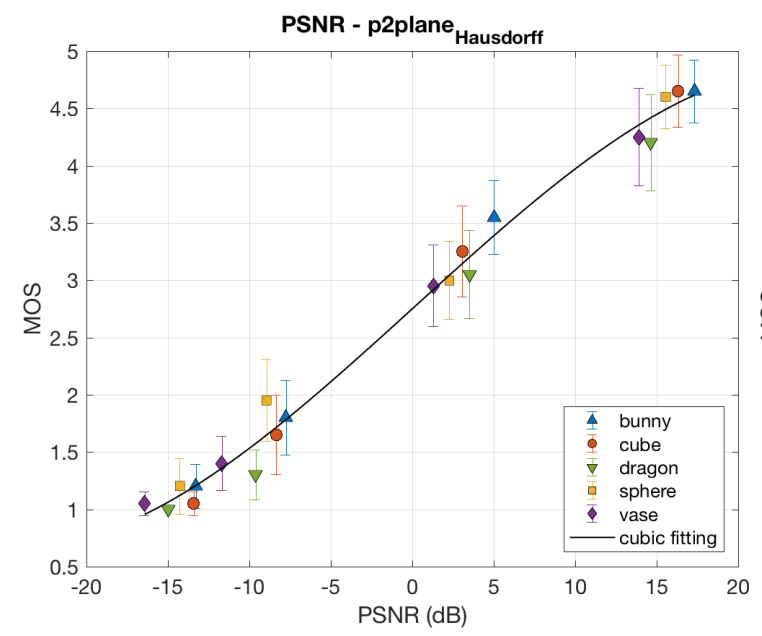

(a) Gaussian noise.

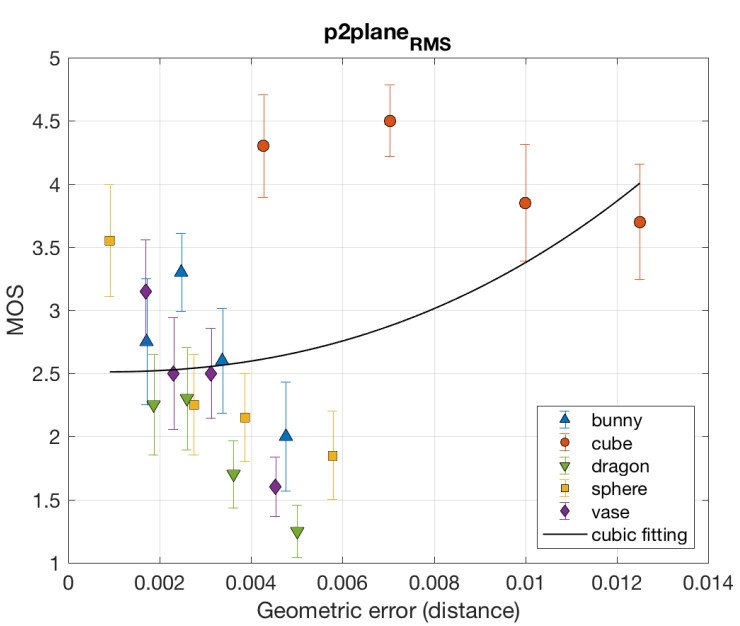

(b) Octree-pruning.

Figure 7: Subjective vs objective results adopting ACR test method.

In both subjective methodologies, strong correlation between the objective metrics and subjective scores can be observed in presence of Gaussian noise. Considering that the objective metrics capture geometrical distances of closest points between the reference and the processed contents, by increasing the standard deviation of noise the objective scores worsen. The subjects were able to perceive such distortions and identify the level of discrepancies, either when the reference was visible or not (i.e., DSIS and ACR, respectively).

On the contrary, the correlation between subjective and objective scores in presence of compression-like distortions is poor. Despite the fact that the level of perceptual impairment is reflected in the objective scores for contents with curved surfaces, this is not the case for objects with planar surfaces, such as cube. As the number of points decreases, less details and more rough representations of curved edges are observed, which has a strong impact on the perceptual quality; thus, the subjects rate the distorted contents lower. This is aligned with our prior knowledge of how the human visual system works, based on which once missing points 
are perceived, people tend to interpolate the visible points. Thus, when the underlying surface is a curve, it is perceived as a straight line which leads to perception of the impairment as more annoying. This is not applied in the case of cube, though, which consists of planar surfaces. In addition, the geometry of this particular content is highly regular, as it was artificially generated. The octree structure, by default, arranges the points of the content in a structured and equally spaced way. Thus, the underlying geometry of cube is not significantly affected and, by extension, the structured loss after octree-pruning is not perceived as truly annoying.

As can be seen in Figures $6 \mathrm{~b}$ and $7 \mathrm{~b}$, cube behaves as an outlier. By removing its scores, the performance of the objective metrics is significantly improved. In Table 4 the performance indexes of the most efficient objective metric per test methodology are provided. In addition, in Figure 8, the scatter plots of the subjective scores against the most efficient objective metric for both test methods, are indicated. These results suggest that the geometrical distances captured by the objective metrics are well aligned with the perceptual quality of distorted objects with curved surfaces.

Table 4: Performance indexes for the most efficient objective metric in octree-pruning without considering cube.

\begin{tabular}{l|cccc|cccc}
\hline \multirow{2}{*}{ Metric } & \multicolumn{4}{|c|}{ DSIS test method } & \multicolumn{4}{c}{ ACR test method } \\
\cline { 2 - 9 } & PCC & SROCC & RMSE & OR & PCC & SROCC & RMSE & OR \\
\hline \multirow{2}{*}{ PSNR - p2point Hausdorff } & 0.9736 & 0.9594 & 0.1520 & 0.0 & 0.8775 & 0.9131 & 0.3002 & 0.3125
\end{tabular}

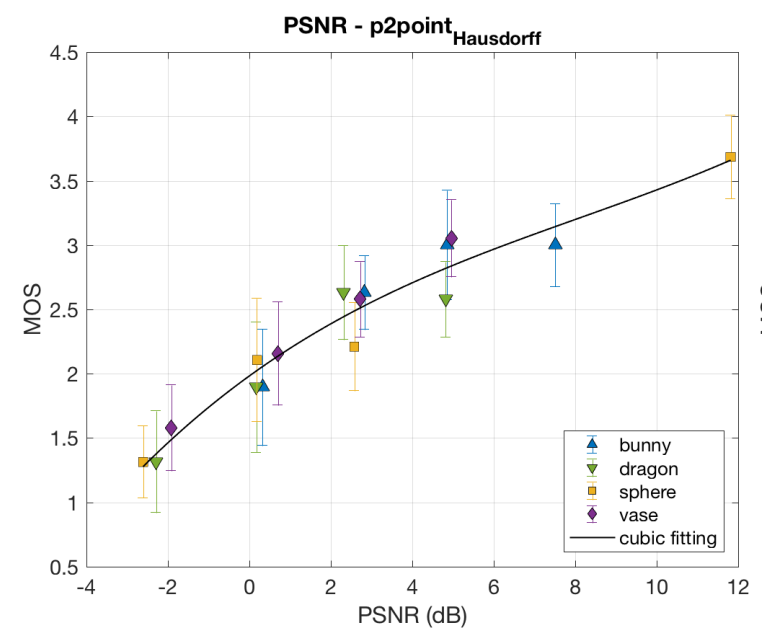

(a) DSIS test method.

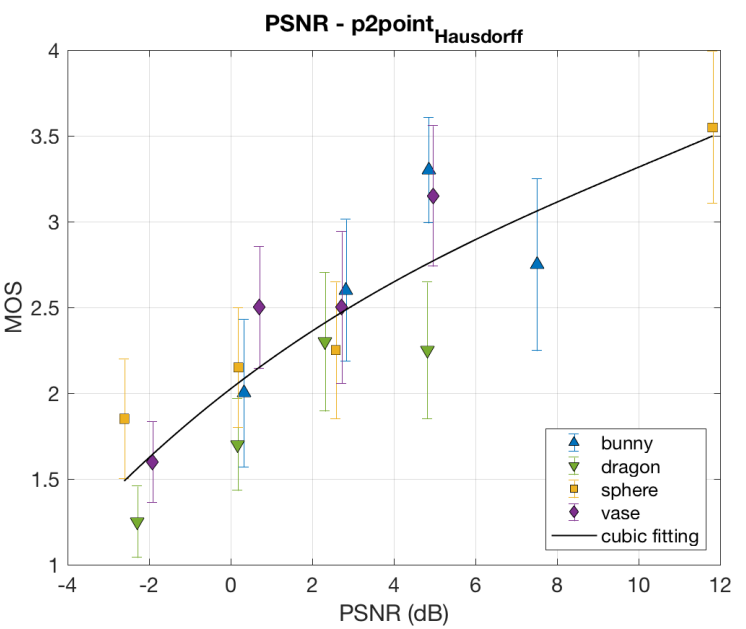

(b) ACR test method.

Figure 8: Subjective vs objective results of octree-pruning without considering cube.

\subsection{Comparison between two subjective methodologies}

In Table 5, the performance indexes of the MOS obtained from the DSIS (i.e., which is considered as ground truth) against the MOS from the ACR subjective methodologies are provided. No fitting, linear and cubic fitting is applied on the scores obtained from the ACR test method.

In Figure 9 the scatter plots comparing the subjective scores of the DSIS against the ACR test methodology are presented. The horizontal and vertical bars indicate the confidence intervals as computed by the scores from the subjective methodology that is reported in the corresponding label. The linear and cubic fitting curves are also included.

In Table 6, the performance indexes of the MOS obtained from the ACR (i.e., which is considered as ground truth) against the MOS from the DSIS subjective methodologies are provided. In this case, in addition to no fitting, a linear and a cubic fitting are applied on the scores obtained from the DSIS test method.

In Figure 10 the scatter plots comparing the subjective scores of the ACR against the DSIS test methods are presented along with the linear and cubic fitting curves. 
Table 5: Performance indexes considering DSIS as ground truth.

\begin{tabular}{|c|c|c|c|c|c|c|c|c|c|}
\hline \multirow{2}{*}{ Fitting } & \multicolumn{9}{|c|}{ Gaussian noise } \\
\hline & $\mathrm{PCC}$ & SROCC & RMSE & OR & $\mathrm{CE}$ & CD & FR & FD & FT \\
\hline No fitting & 0.9920 & 0.9763 & 0.2109 & 0.20 & $100 \%$ & $95.79 \%$ & $0 \%$ & $1.58 \%$ & $2.63 \%$ \\
\hline Linear fitting & 0.9920 & 0.9763 & 0.1748 & 0.10 & $100 \%$ & $95.79 \%$ & $0 \%$ & $1.58 \%$ & $2.63 \%$ \\
\hline Cubic fitting & 0.9921 & 0.9763 & 0.1738 & 0.05 & $100 \%$ & $95.79 \%$ & $0 \%$ & $1.58 \%$ & $2.63 \%$ \\
\hline \multirow{2}{*}{ Fitting } & \multicolumn{9}{|c|}{ Octree-pruning } \\
\hline & $\mathrm{PCC}$ & SROCC & RMSE & OR & $\mathrm{CE}$ & $\mathrm{CD}$ & FR & $\overline{F D}$ & FT \\
\hline No fi & 0.9478 & 0.9551 & 0.3049 & 0.20 & $100 \%$ & $84.74 \%$ & $0 \%$ & $8.94 \%$ & $6.32 \%$ \\
\hline Linear fitting & 0.9478 & 0.9551 & 0.3035 & 0.20 & $100 \%$ & $84.74 \%$ & $0 \%$ & $7.89 \%$ & $7.37 \%$ \\
\hline Cubic fitting & 0.9520 & 0.9551 & 0.2913 & 0.20 & $100 \%$ & $86.84 \%$ & $0 \%$ & $8.42 \%$ & $4.74 \%$ \\
\hline
\end{tabular}

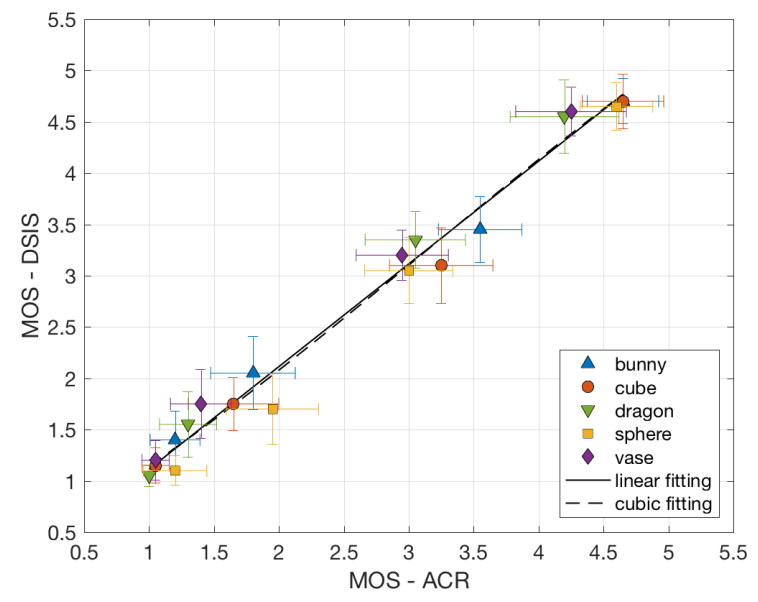

(a) Gaussian noise.

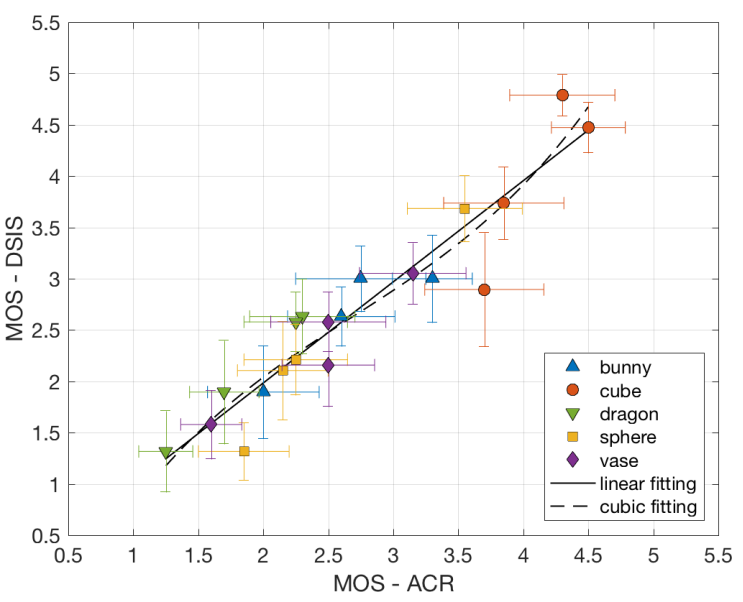

(b) Octree-pruning.

Figure 9: Comparison between subjective methodologies, assuming DSIS method as ground truth.

Table 6: Performance indexes considering ACR as ground truth.

\begin{tabular}{|c|c|c|c|c|c|c|c|c|c|}
\hline \multirow{2}{*}{ Fitting } & \multicolumn{9}{|c|}{ Gaussian noise } \\
\hline & $\mathrm{PCC}$ & SROCC & RMSE & OR & $\mathrm{CE}$ & CD & FR & FD & FT \\
\hline No fi & 0.9920 & 0.9763 & 0.2109 & 0.25 & $100 \%$ & $95.79 \%$ & $0 \%$ & $2.63 \%$ & $1.58 \%$ \\
\hline Lin & 920 & 3 & 0.1 & 0.10 & $100 \%$ & $95.79 \%$ & $0 \%$ & $2.63 \%$ & $1.58 \%$ \\
\hline & 922 & 0 & 0.1 & 0.10 & $100 \%$ & $95.79 \%$ & $0 \%$ & $1.58 \%$ & $2.63 \%$ \\
\hline \multirow{2}{*}{ Fitti } & \multicolumn{9}{|c|}{ 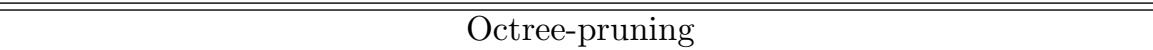 } \\
\hline & $\mathrm{PCC}$ & $\overline{\mathrm{CC}}$ & RMSE & OR & $\mathrm{CE}$ & $\mathrm{CD}$ & FR & FD & FT \\
\hline & 9478 & & 0.3049 & 0.15 & $100 \%$ & $84.74 \%$ & $0 \%$ & $6.32 \%$ & $8.94 \%$ \\
\hline & 0.9478 & 0.9551 & 0.2917 & 0.20 & $100 \%$ & $83.68 \%$ & $0 \%$ & $4.74 \%$ & $11.58 \%$ \\
\hline Cubic fitting & 0.9538 & 0.9551 & 0.2747 & 0.10 & $100 \%$ & $83.16 \%$ & $0 \%$ & $4.21 \%$ & $12.63 \%$ \\
\hline
\end{tabular}

Based on our results for Gaussian noise, in both Figures 9a and 10a the fitting curve achieves an angle of $45^{\circ}$. However, the intercept is not zero. This, essentially, means that DSIS methodology gives slightly higher MOS results consistently. This tendency can be explained by the fact that visual quality of point clouds is difficult to be assessed considering that, essentially, a collection of points is displayed and "holes" in the contents are always visible. When the reference point cloud is absent, which enables rating according to the actual visual quality and not based on relative differences, it is reasonable to obtain lower scores.

Considering the performance indexes, it is clear that the subjective methodologies are strongly correlated. The low RMSE and OR values, are evident for high accuracy and consistency of the results. Furthermore, the $\mathrm{CE}$ is $100 \%$, indicating that there is no statistically significant difference between the MOS obtained from each methodology. This is also why OE and UE are not indicated in the tables. There is $0 \% \mathrm{FR}$, which is the most 


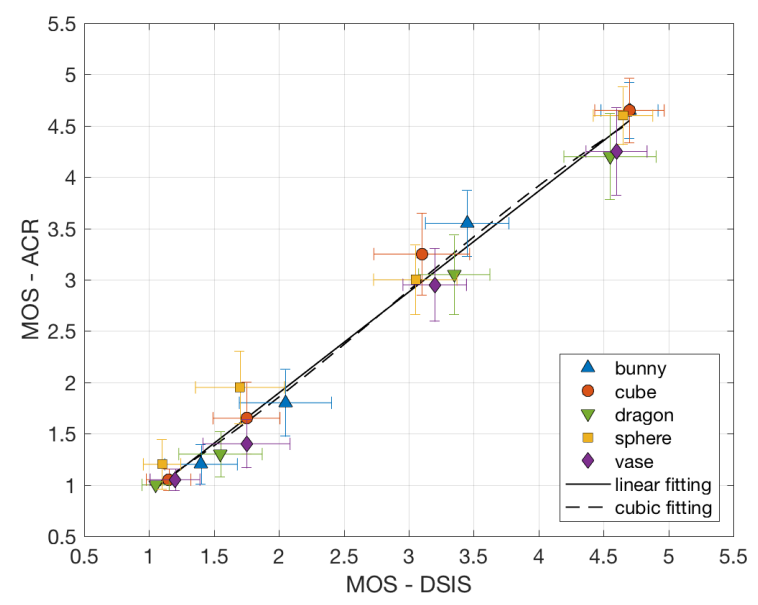

(a) Gaussian noise.

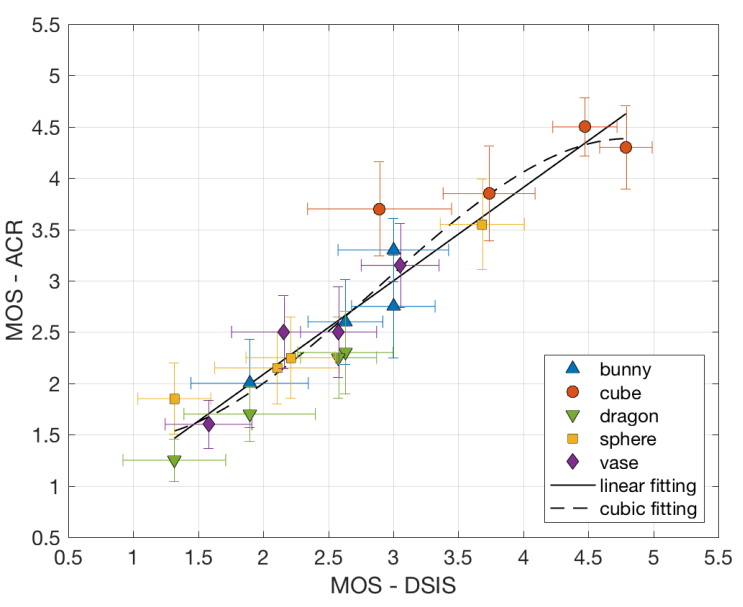

(b) Octree-pruning.

Figure 10: Comparison between subjective methodologies, assuming ACR method as ground truth.

offensive error, while the FD and FT have very low percentages.

Based on our results after octree-pruning, when the MOS results obtained from DSIS are considered as ground truth, an angle of $45^{\circ}$ can be observed in the fitting line. However, when the ACR experiment is set as the ground truth, the subjective scores from ACR are slightly higher for higher levels and slightly lower for lower levels of impairement, leading to an angle less than $45^{\circ}$.

The linearity and monotonicity coefficients are high in both cases while the RMSE and OR values are still relatively low. Moreover, the $\mathrm{CE}$ is $100 \%$ and the FR is $0 \%$. The high FD percentages observed in Table 5 when setting the DSIS scores as ground truth suggest that this methodology does not differentiate two stimuli, while the ACR methodology decides that they are statistically different. The same tendency can be observed through the high FT percentages of Table 6. In other terms, the ACR methodology shows more discriminating power for such type of distortion when compared to the DSIS.

One-way ANOVA was performed on the processed contents grouped by methodology, separately for each degradation type. Results showed that there is no significant difference between the scores obtained with either DSIS or ACR, neither for Gaussian noise $(p=0.2711)$ nor for octree-pruning $(p=0.7598)$. One-way ANOVA was also performed on the entire set of processed contents. Results show that the two methodologies are statistically equivalent $(p=0.5086)$.

To gain further insights on what impact different parameters had on the final scores, multi-way ANOVA was performed on the data associated with each degradation type, separately for level of impairment and content. Since the type of methodology was shown not to bear any statistically significant difference, the results were not divided by methodology.

Multi-way ANOVA performed on data from Gaussian noise shows that both contents and degradation levels present statistical differences ( $p=0$ in both cases), while the interaction did not have an impact on the final scores $(p=0.4291)$. In order to better understand from which specific parameter the differences arose, we performed one-way ANOVA on the same set of data, separately for level of impairment and content. As expected, the level of impairments were found to be significantly different for all contents. However, analysis on different degradation levels showed that, while the contents give statistically different results with the highest levels of degradation ( $p=0.0108$ and $p=0.0188$ for $\sigma=0.008$ and $\sigma=0.016$, respectively), they are statistically equivalent for the lowest levels of degradation ( $p=0.1476$ and $p=0.0965$ for $\sigma=0.0005$ and $\sigma=0.002$, respectively). This can be explained by the fact that the dragon was rated lower than the other contents in the ACR test methodology, as explained in Section 5.2, and for $\sigma=0.008$ statistical difference was found. In addition, bunny was rated higher than the other contents for $\sigma=0.016$ in the context of the DSIS test method, leading to statistical significant difference. In this case, no particular reason is obvious - maybe the content was pleasant to the subjects. 
Multi-way ANOVA performed on the data after octree-pruning also shows that degradation levels and contents have statistical differences ( $p=0$ in both cases), and the interaction was also found to be statistically significant for the final scores $(p=0)$. One-way ANOVA performed separately for each content showed that the level of impairments gave statistically different results $(p=0)$, as expected. Finally, performing one-way ANOVA on different levels of impairment showed statistical difference among contents for all levels $(p=0)$. This can be explained, as cube was rated remarkably higher than all the other contents, for both cases of DSIS and ACR test methods.

\section{CONCLUSIONS}

In this paper a subjective methdology closer to realistic visualization of point cloud content was proposed and evaluated. Our results show that although point cloud is a modality difficult to be assessed in terms of visual quality, strong correlation is achieved with respect to a test method that allows the simultaneous visualization of the reference content. In particular, based on our analysis, ACR and DSIS methodologies found to be statistically equivalent. The comparison between DSIS and ACR methodology shows that in the first case, the subjects tend to rate the contents slightly higher in the presence of Gaussian noise, while for octree-pruning, no repetitive tendencies are observed. The DSIS methodology is more consistent in terms of identifying the level of impairment, which is also projected in the trends of the MOS against degradation values curves. However, subjects tend to rate based on relative differences (i.e., geometrical distances or number of points) and not explicitly according to the visual quality; the latter is achieved through the ACR methodology though. For instance, adopting the ACR test method, dragon and vase are rated lower than the other contents in the presence of Gaussian noise, due to complexity and geometrical structure, respectively. The classification errors, also, show that the ACR methodology shows more discriminating power when compared to the DSIS for octreepruning. However, larger confidence intervals are observed, suggesting the necessity of involving more subjects in the experiments. Regarding the performance of the objective metrics with respect to the subjective scores, in both test methodologies, similar trends are observed; that is, in the presence of Gaussian noise strong correlation is achieved, while after octree-pruning, regular objects with planar surfaces behave as outliers and the correlation is poor. The DSIS method, though, provides better results for each type of impairment (i.e., if we exclude the scores of cube from octree-pruning).

\section{ACKNOWLEDGMENT}

This paper reports a research performed under the frame- work of a collaborative project Random Access Video En- coding (RAVE) with Teleport SA and funded by The Swiss Commission for Technology and Innovation (CTI) under the grant 18875.1PFES-ES. A special thanks to Irene Viola and Evgeniy Upenik for the fruitful discussions. 


\section{REFERENCES}

[1] MPEG Virtual Reality AhG, "Summary of MPEG VR questionnaire results." ISO/IEC JTC N16542 (Oct. 2016).

[2] Smisek, J., Jancosek, M., and Pajdla, T., "3D with Kinect," in [Consumer Depth Cameras for Computer Vision], pp. 3-25, Springer London (2013).

[3] Matsuda, N., Cossairt, O., and Gupta, M., "MC3d: Motion contrast 3d scanning," in [Computational Photography (ICCP), 2015 IEEE International Conference on], pp. 1-10 (2015).

[4] Newcombe, R. A. and Davison, A. J., "Live dense reconstruction with a single moving camera," in [Computer Vision and Pattern Recognition (CVPR), 2010 IEEE Conference on], 1498-1505, IEEE (Aug. 2010).

[5] Newcombe, R. A., Izadi, S., Hilliges, O., Molyneaux, D., Kim, D., Davison, A. J., Kohi, P., Shotton, J., Hodges, S., and Fitzgibbon, A., "KinectFusion: Real-time dense surface mapping and tracking," in [Mixed and augmented reality (ISMAR), 2011 10th IEEE international symposium on], 127-136, IEEE (2011).

[6] Newcombe, R. A., Lovegrove, S. J., and Davison, A. J., "DTAM: Dense tracking and mapping in real-time," in [Computer Vision (ICCV), 2011 IEEE International Conference on], 2320-2327, IEEE (2011).

[7] Engel, J., Schöps, T., and Cremers, D., "LSD-SLAM: Large-scale direct monocular SLAM," in [European Conference on Computer Vision], 834-849, Springer (2014).

[8] Dai, A., Nießner, M., Zollhöfer, M., Izadi, S., and Theobalt, C., "BundleFusion: Real-Time Globally Consistent 3d Reconstruction Using On-the-Fly Surface Reintegration," ACM Trans. Graph. 36, 24:1-24:18 (May 2017).

[9] Hoppe, H., "Efficient implementation of progressive meshes," Computers $\& 3$ Graphics 22, 27-36 (Feb. 1998).

[10] Gumhold, S. and Straßer, W., "Real time compression of triangle mesh connectivity," in [Proceedings of the 25th Annual Conference on Computer Graphics and Interactive Techniques], SIGGRAPH '98, 133-140, ACM (1998).

[11] Rossignac, J., "Edgebreaker: connectivity compression for triangle meshes," IEEE Transactions on Visualization and Computer Graphics 5, 47-61 (Jan. 1999).

[12] Peng, J., Kim, C.-S., and Jay Kuo, C. C., "Technologies for 3d mesh compression: A survey," Journal of Visual Communication and Image Representation 16, 688-733 (Dec. 2005).

[13] Han, S. R., Yamasaki, T., and Aizawa, K., "Time-Varying Mesh Compression Using an Extended Block Matching Algorithm," IEEE Transactions on Circuits and Systems for Video Technology 17, 1506-1518 (Nov. 2007).

[14] ITU-R BT.500-13, "Methodology for the subjective assessment of the quality of television pictures." International Telecommunication Union (Jan. 2012).

[15] Alexiou, E. and Ebrahimi, T., "On subjective and objective quality evaluation of point cloud geometry," in [Proceedings of the 9th International Conference on Quality of Multimedia Experience (QoMEX)], (2017).

[16] Alexiou, E., Upenik, E., and Ebrahimi, T., "Towards subjective quality assessment of point cloud imaging in augmented reality," in [2017 IEEE 19th International Workshop on Multimedia Signal Processing (MMSP)], (2017).

[17] Zhang, J., Huang, W., Zhu, X., and Hwang, J. N., "A subjective quality evaluation for 3d point cloud models," in [International Conference on Audio, Language and Image Processing], 827-831 (2014).

[18] Mekuria, R., Blom, K., and Cesar, P., "Design, Implementation, and Evaluation of a Point Cloud Codec for Tele-Immersive Video," IEEE Transactions on Circuits and Systems for Video Technology 27, 828-842 (Apr. 2017).

[19] Javaheri, A., Brites, C., Pereira, F., and Ascenso, J., "Subjective and objective quality evaluation of 3D point cloud denoising algorithms." ISO/IEC JTC m75024 (Mar. 2017).

[20] Girardeau-Montaut, D., "Cloud compare -3d point cloud and mesh processing software."

[21] Peng, J. and Kuo, C. J., "Octree-based progressive geometry encoder," in [ITCom], 301-311, International Society for Optics and Photonics (2003).

[22] Huang, Y., Peng, J., Kuo, C.-C. J., and Gopi, M., "Octree-based progressive geometry coding of point clouds," in $[S P B G], 103-110$ (2006).

[23] Schnabel, R. and Klein, R., "Octree-based point-cloud compression," in [Spbg], 111-120 (2006). 
[24] Yan Huang, Jingliang Peng, Kuo, C.-C., and Gopi, M., "A generic scheme for progressive point cloud coding," IEEE Transactions on Visualization and Computer Graphics 14(2), 440-453 (2008).

[25] Kammerl, J., Blodow, N., Rusu, R. B., Gedikli, S., Beetz, M., and Steinbach, E., "Real-time compression of point cloud streams," in [2012 IEEE International Conference on Robotics and Automation], 778-785 (2012).

[26] Rusu, R. B. and Cousins, S., "3D is here: Point cloud library (PCL)," in [2011 IEEE International Conference on Robotics and Automation], 1-4 (2011).

[27] ITU-T P.1401, "Methods, metrics and procedures for statistical evaluation, qualification and comparison of objective quality prediction models." International Telecommunication Union (Jul. 2012).

[28] Tian, D., Ochimizu, H., Feng, C., Cohen, R., and Vetro, A., "Evaluation metrics for point cloud compression." ISO/IEC JTC m74008 (January 2017).

[29] ITU-T J.149, "J.149 : Method for specifying accuracy and cross-calibration of Video Quality Metrics (VQM)." International Telecommunication Union (Mar. 2004). 